\title{
Free and forced vibrations of the shells of revolution interacting with the liquid
}

\author{
E. Strelnikova, E. Yeseleva, V. Gnitko \& V. Naumenko \\ Institute for Mechanical Engineering Problems of the Ukrainian \\ Academy of Sciences, Ukraine
}

\begin{abstract}
The coupled problem of free and forced vibration of shells of revolution interacting with the liquid is under consideration. The problem was reduced to singular integral equations. The main objective of this paper is to present a detailed study of free and forced vibrations of a shell of revolution with an arbitrary meridian. For solving the boundary value problem of determining the fluid pressure on the shell, the boundary element method (BEM) is used. This method substantially reduces the computer time for the analysis and reveals new qualitative possibilities in modeling the dynamic behavior of shells. Numerical investigations of natural frequencies and mode shapes of the cylindrical tank with the incompressible fluid have been carried out. The behavior of structures subjected to a dynamic loading and interacting with the fluid is investigated.
\end{abstract}

Keywords: free vibrations, forced vibrations, fluid-shell interaction, boundary and finite element methods.

\section{Introduction}

Fluid-structure interaction has been studied extensively in the last decade [1-4]. The urgency of the problem is connected with wide application of the liquid storage tanks in engineering. The liquid storage tanks are important components of lifeline and industrial facilities. They are critical elements in municipal water supply and fire fighting systems, and in many industrial facilities for storage of water, oil, chemicals and liquefied natural gas, etc.

Several complexities are involved in the strength analysis of such tanks. If the excitation frequency is near the natural one, the high dynamic pressures may be produced on the tank walls due to the resonance. So calculation of the natural 
frequencies and mode shapes is of great importance. Containers partially filled with the liquid are often subjected to an intensive external excitation. Problems associated with the dynamic behavior of the liquid storage tanks involve the analysis of two systems: the tank and the liquid, as well as the interaction between them along their boundaries. Due to the complexity of the problem, most of the original studies were experimental in nature. Several simplified theoretical investigations were also conducted and a few of these studies have been used as a basis for current design standards.

In $[5,6]$ authors offer the approach based on using the boundary element method (BEM) to the problem of natural vibrations of the fluid-filled elastic shells of revolution filled by the liquid, as well as to the problem of natural liquid vibrations in the rigid vessels. This approach has the certain advantages. In the basic equations the functions and their derivatives will be defined on the domain boundaries only. That allows reducing per unit dimension of the problem. In the present work the BEM technique is developed to research the processes of fluidstructure interaction of the shells of revolution at the dynamic loading. In the present paper the new effective method is elaborated. This method gives new qualitative possibilities in modeling the dynamic coupled problems.

\section{Problem statement}

Let us consider the problem of free and forced vibrations of an elastic shell of revolution with an arbitrary meridian and partially filled with the ideal incompressible fluid. We shall indicate a moistened surface of a shell through $S_{1}$. Let refer the Cartesian coordinate system 0xyz connected with a shell. The free surface of the liquid $S_{0}$ coincides with the plane $x 0 y$ in unperturbed state.

Suppose that the fluid is ideal and incompressible and the flow induced by vibrations of the shell is irrotational and consider small shell and fluid vibrations. Let $\boldsymbol{U}$ is the vector-function of the shell displacements and $\boldsymbol{V}$ is the fluid velocity vector.

Under these suppositions, there exists a velocity potential $\varphi$ defined as

$$
V_{x}=\frac{\partial \varphi}{\partial x}, \quad V_{y}=\frac{\partial \varphi}{\partial y}, \quad V_{z}=\frac{\partial \varphi}{\partial z} .
$$

Assuming that the natural velocity of the fluid is zero, the pressure value, according to the Cauchy-Lagrange integral, can be represented as follows

$$
P_{l}=-\rho_{l} \frac{\partial \varphi}{\partial t} .
$$

Here $P_{l}$ is hydrodynamic pressure in the disturbed motion, and $\rho_{l}$ is the fluid density. A system of governing equations of motion of elastic shell with the liquid in the operator form is given by

$$
\boldsymbol{L} \boldsymbol{U}+\boldsymbol{M} \ddot{\boldsymbol{U}}=P_{l}+Q,
$$

where $\boldsymbol{L}, \boldsymbol{M}$ are operators of elastic and mass forces of the shell; $\boldsymbol{U}=\left(u_{1}, u_{2}, w\right)$ is the displacement vector; $Q(t)$ is the vector of external surface load. 
The kinematical boundary condition of the continuous fluid motion on the wetted shell surface $S_{1}$ can be represented as follows:

$$
\boldsymbol{V} \boldsymbol{n}=\dot{\boldsymbol{U}} \boldsymbol{n}=\dot{w},
$$

where $\boldsymbol{n}$ is an external unit normal to wetted surface of the shell and $w$ is the shell normal displacement.

Let $z=H$ is the coordinate of non-disturbed free surface $S_{0}$ of the fluid. The boundary condition on $S_{0}$ corresponds to the requirement of pressure absence on the free surface. It can be written as follows:

$$
P_{l}=0 .
$$

The hydrodynamic problem (1)-(4) can be described by using the only unknown function $\varphi(x, y, z, t)$. To determine the velocity potential we obtain the following boundary value problem:

$$
\begin{gathered}
\boldsymbol{L U}+\boldsymbol{M} \ddot{\boldsymbol{U}}+\rho \dot{\varphi}=Q, \\
\nabla^{2} \varphi=0, \\
\frac{\partial \varphi}{\partial n}=\dot{w}, P \in S_{1}, \\
\dot{\varphi}=0, P \in S_{0} .
\end{gathered}
$$

Here it is supposed, that $S=S_{1} \cup S_{0}$ and point $P$ belongs to surface $S$.

\section{System of the boundary integral equations}

To solve the coupled hydro-elasticity problem it is necessary to determine the fluid pressure on the wetted surface of the shell. This problem is here reduced to the solution of the system of singular integral equations. In this section it is supposed, that velocity of the elastic shell is given. Determination of the fluid pressure on the wetted surface of the shell leads to the mixed boundary value problem for the Laplace equations (5)-(8).

We will seek the natural modes of vibration in the fluid in the form

$$
\begin{aligned}
& U(x, y, z, t)=\sum_{k=1}^{m} u_{k}(x, y, z) c_{k}(t), \\
& \varphi(x, y, z, t)=\sum_{k=1}^{m} \varphi_{k}(x, y, z) \dot{c}_{k}(t) .
\end{aligned}
$$

Here functions $u_{k}(x, y, z)$ are modes of natural vibrations in vacuum, $c_{k}(t)$ are unknown factors. To determine the set of functions $\varphi_{k}$ the following boundary value problems are formulated:

$$
\begin{gathered}
\nabla^{2} \varphi_{k}=0, \frac{\partial \varphi_{k}}{\partial n}=w_{k}, P_{0} \in S_{1}, \\
\varphi_{k}=0, P_{0} \in S_{0}, \quad k=1, m .
\end{gathered}
$$


We will seek the harmonic functions $\varphi_{k}$ in the form of the sum of simple and double layer potentials [7]. The direct formulation of boundary integral equation method is in use. Dropping the index $k$, we have

$$
2 \pi \varphi\left(P_{0}\right)=\iint_{S} \frac{\partial \varphi}{\partial n} \frac{1}{\left|P-P_{0}\right|} d S-\iint_{S} \varphi \frac{\partial}{\partial n} \frac{1}{\left|P-P_{0}\right|} d S .
$$

For the mixed problem described by eqns (5)-(8) this integral representation leads to the following system of singular integral equations:

$$
\begin{gathered}
2 \pi \varphi\left(P_{0}\right)+\iint_{S_{1}} \varphi \frac{\partial}{\partial n} \frac{1}{\left|P-P_{0}\right|} d S_{1}-\iint_{S_{0}} q \frac{1}{\left|P-P_{0}\right|} d S_{0}= \\
=\iint_{S_{1}} w \frac{1}{\left|P-P_{0}\right|} d S_{1}, \quad P_{0} \in S_{1}, \\
\iint_{S_{1}} \varphi \frac{\partial}{\partial n} \frac{1}{\left|P-P_{0}\right|} d S_{1}-\iint_{S_{0}} q \frac{1}{\left|P-P_{0}\right|} d S_{0}=\iint_{S_{1}} w \frac{1}{\left|P-P_{0}\right|} d S_{1}, \quad P_{0} \in S_{0},
\end{gathered}
$$

with regard to unknown functions $\varphi$ and $q$. Here function $\varphi$ defined on the surface $S_{1}$, represents the pressure on the wetted surface of the shell, and function $q$, defined on the surface $S_{0}$, is the normal component of the fluid velocity on the free surface.

We use furthermore the cylindrical coordinate system and represent unknown functions as Fourier series by circumferential coordinate

$$
w=w(r, z) \cos \alpha \theta, \quad \varphi=\varphi(r, z) \cos \alpha \theta .
$$

We will provide transformation in the same way as in [7]. The purpose is to bring the kernels in eqn (13) to standard elliptic integrals. Since

$$
\begin{gathered}
\left|P-P_{0}\right|=\sqrt{r^{2}+r_{0}^{2}+\left(z-z_{0}\right)^{2}-2 r r_{0} \cos \left(\theta-\theta_{0}\right)}, \\
a=r^{2}+r_{0}^{2}+\left(z-z_{0}\right)^{2}, \quad b=2 r r_{0},
\end{gathered}
$$

we obtain the following expression

$$
\frac{\partial}{\partial n} \frac{1}{\left|P-P_{0}\right|}-\frac{\left(\overrightarrow{P-P_{0}}\right) \cdot \vec{n}}{\left|P-P_{0}\right|^{3}}=-\frac{n_{r}\left[r-r_{0} \cos \left(\theta-\theta_{0}\right)\right]+n_{z}\left(z-z_{0}\right)}{\left(\sqrt{a-b \cos \left(\theta-\theta_{0}\right.}\right)^{3}} .
$$

As the result of these transformations we have obtained the system of integral equations with one-dimensional integrals computed only along the meridian of the wetted surface of the shell and radius of the fluid free surface.

This system takes the following form

$$
2 \pi \varphi\left(z_{0}\right)+\int_{\Gamma} \varphi(z) Q\left(z, z_{0}\right) r(z) d \Gamma-\int_{0}^{R} q(\rho) \Psi\left(P, P_{0}\right) \rho d \rho=
$$




$$
\begin{gathered}
=\int_{\Gamma} w(z) \Psi\left(P, P_{0}\right) r(z) d \Gamma_{1}, \quad P_{0} \in S_{1}, \\
\int_{\Gamma} \varphi(z) Q\left(z, z_{0}\right) r(z) d \Gamma-\int_{0}^{R} q(\rho) \Psi\left(P, P_{0}\right) \rho d \rho=\int_{\Gamma} w(z) \Psi\left(P, P_{0}\right) r(z) d \Gamma_{1}, \quad P_{0} \in S_{0} .
\end{gathered}
$$

Here

$$
\begin{gathered}
Q\left(z, z_{0}\right)=\frac{4}{\sqrt{a+b}}\left\{\frac{1}{2 r}\left[\frac{r^{2}-r_{0}^{2}+\left(z_{0}-z\right)^{2}}{a-b} \mathrm{E}_{\alpha}(k)-\mathrm{F}_{\alpha}(k)\right] n_{r}+\frac{z_{0}-z}{a-b} \mathrm{E}_{\alpha}(k) n_{z}\right\}, \\
\Psi\left(P, P_{0}\right)=\frac{4}{\sqrt{a+b}} \mathrm{~F}_{\alpha}(k) .
\end{gathered}
$$

Here the following notations are introduced

$$
\begin{aligned}
& \mathrm{E}_{\alpha}(k)=(-1)^{\alpha}\left(1-4 \alpha^{2}\right)^{\pi / 2} \cos 2 \alpha \psi \sqrt{1-k^{2} \sin ^{2} \psi} d \psi, \\
& \mathrm{F}_{\alpha}(k)=(-1)^{\alpha} \int_{0}^{\pi / 2} \frac{\cos 2 \alpha \psi d \psi}{\sqrt{1-k^{2} \sin ^{2} \psi}}, \quad k^{2}=\frac{2 b}{a+b} .
\end{aligned}
$$

Letting $\alpha=0$ in the above expression, we obtain the standard elliptic first and second kind integrals.

For numerical solution of system described by eqns (13) the boundary element method with constant approximation of unknown density on elements was used.

\section{The mode superposition method for coupled dynamic problems}

After definition of functions $\varphi_{k}$ we substitute expressions (9), (10) in eqn (5) and obtain the following equation

$$
\boldsymbol{L}\left(\sum_{k=1}^{m} c_{k} u_{k}\right)+\boldsymbol{M}\left(\sum_{k=1}^{m} \ddot{c}_{k} u_{k}\right)=-\rho \sum_{k=1}^{m} \ddot{c}_{k} \varphi_{k}+Q .
$$

Let us $\omega_{k}, u_{k}$ are natural frequencies and free vibrations mode shapes of the shell in vacuum. For these the following relationships are valid

$$
\boldsymbol{L} u_{k}=\omega_{k}^{2} \boldsymbol{M} u_{k},\left(\boldsymbol{M} u_{k}, u_{j}\right)=\delta_{k j} .
$$


Considering the result of dot product of eqn (15) by $u_{j}$ and taking into account relationship (16), we come to the set of second order differential equations about unknown factors $c_{k}$

$$
\omega_{j}^{2} \delta_{k j} c_{j}+\delta_{k j} \ddot{c}_{j}=-\rho \sum_{k=1}^{m} \ddot{c}_{k} \varphi_{k} u_{j}+Q u_{j}, \quad j=1, m .
$$

To solve this system the Runge-Kutta method was used. Natural frequencies and modes of the shell vibrations in vacuum were defined by the finite element method.

\section{Numerical results}

Let us consider a cylindrical shell with a flat bottom partially filled with the fluid. The geometry of the tank is shown in Figure 1 and the parameters are following: the radius is $R=1 \mathrm{~m}$, the thickness is $h=0.01 \mathrm{~m}$, the length $L=2 \mathrm{~m}$, Young's modulus $E=2 \cdot 10^{5} \mathrm{MPa}$, Poisson's ratio $v=0.3$, the material's density is $\rho=7800 \mathrm{~kg} / \mathrm{m}^{3}$, the fluid density $\rho_{l}=1000 \mathrm{~kg} / \mathrm{m}^{3}$. The filling level of the fluid is denoted as $H$. Boundary conditions are following: $u_{r}=u_{z}=u_{\theta}=0$ to $z=0$ and $r=R$.

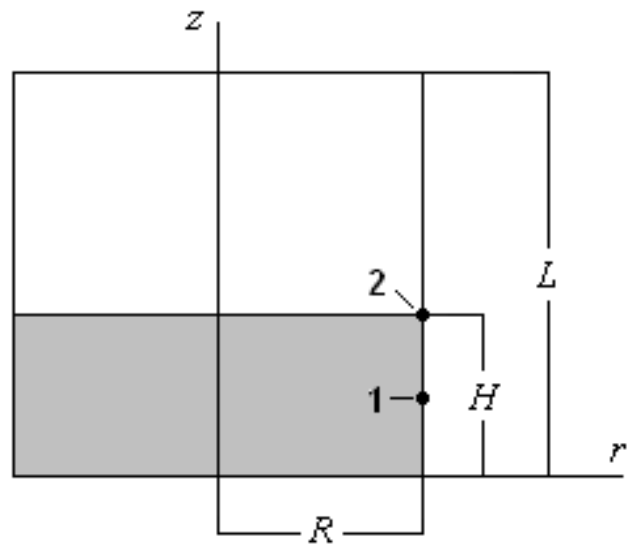

Figure 1: Cylindrical tank with the fluid.

At first we analyze free vibrations of the empty shell and the shell with the liquid. In the case of free vibrations we have $Q=0$ in eqns (17). The results obtained for different numbers of circumferential waves $\alpha$ are presented in Table 1. Here the natural frequencies calculated by proposed reduced method and finite element method (FEM) are demonstrated. Our results showed good agreement with ones obtained by usage the finite element complex. 
Table 1: $\quad$ Natural frequencies of cylindrical tank, $H=0.8 \mathrm{~m}$.

\begin{tabular}{|c|c|c|c|c|}
\hline \multirow{2}{*}{$\alpha$} & \multirow{2}{*}{$m$} & \multicolumn{3}{|c|}{ Natural frequencies, Hz } \\
\cline { 3 - 5 } & & \multirow{2}{*}{ Empty tank } & \multicolumn{2}{|c|}{ Fluid-filled tank } \\
\cline { 3 - 5 } & & 23.23 & 8.15 & Presented method \\
\hline \multirow{2}{*}{0} & 1 & 91.10 & 45.04 & 4.06 \\
\cline { 2 - 5 } & 2 & 48.52 & 20.98 & 20.86 \\
\hline \multirow{2}{*}{1} & 1 & 145.30 & 77.28 & 77.04 \\
\cline { 2 - 5 } & 2 & 79.77 & 40.04 & 39.90 \\
\hline \multirow{2}{*}{2} & 1 & 117.07 & 109.51 & 108.89 \\
\cline { 2 - 5 } & 2 & \multicolumn{3}{|c}{} \\
\hline
\end{tabular}

The next example is connected with forced vibrations coupled problem for cylindrical tank practically filled with the fluid. The radial load is suddenly applied to cylindrical surface of the tank $Q(t)=Q_{0} T(t)$, where $Q_{0}=10 \mathrm{MPa}-$ distributed pressure, $T(t)=\exp (-t / \tau), \tau=14.2 \cdot 10^{-6} \mathrm{~s}$.

Figures 2 and 3 are representative of the forced motion response as calculated by proposed method - solid lines and by the finite element complex - dash lines. Figure 2 shows the radial displacement response calculated in point 1 and Figure 3 shows the same one calculated in point 2 . The point 1 is situated in the wetted part of the wall whereas point 2 belongs to boundary of the liquid free surface.

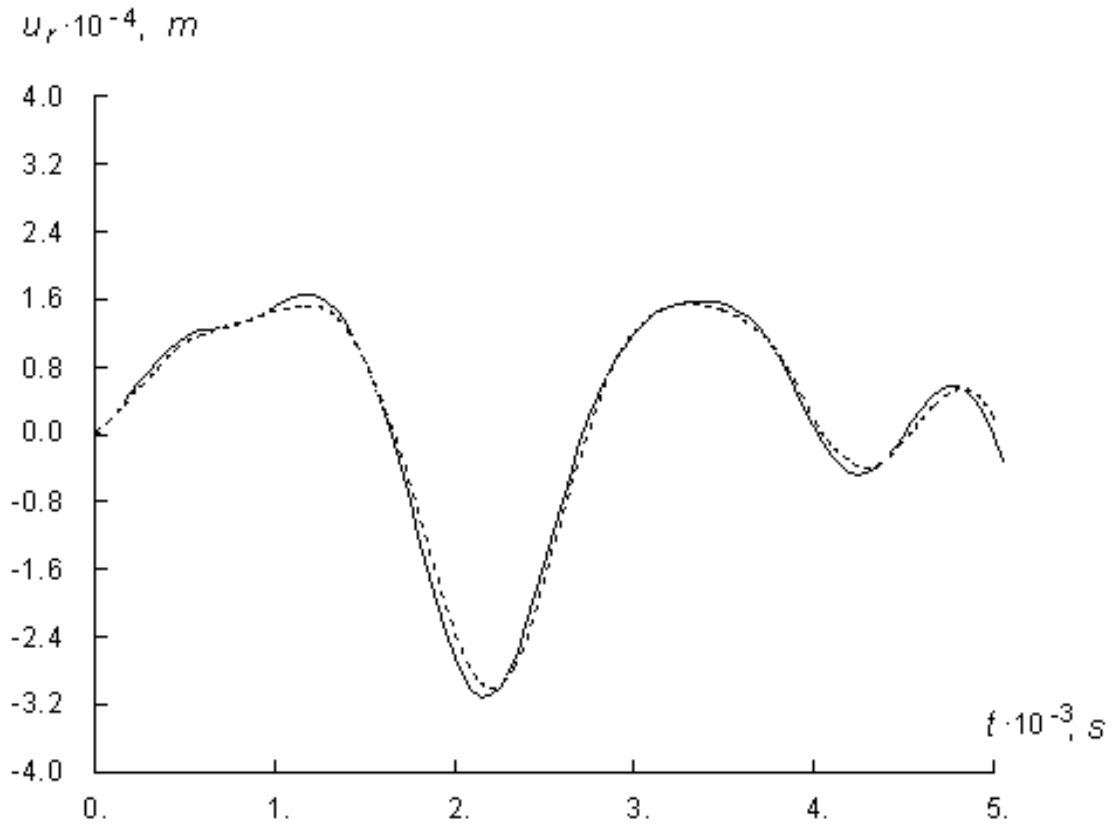

Figure 2: $\quad$ Time history of the radial displacement at point 1. 


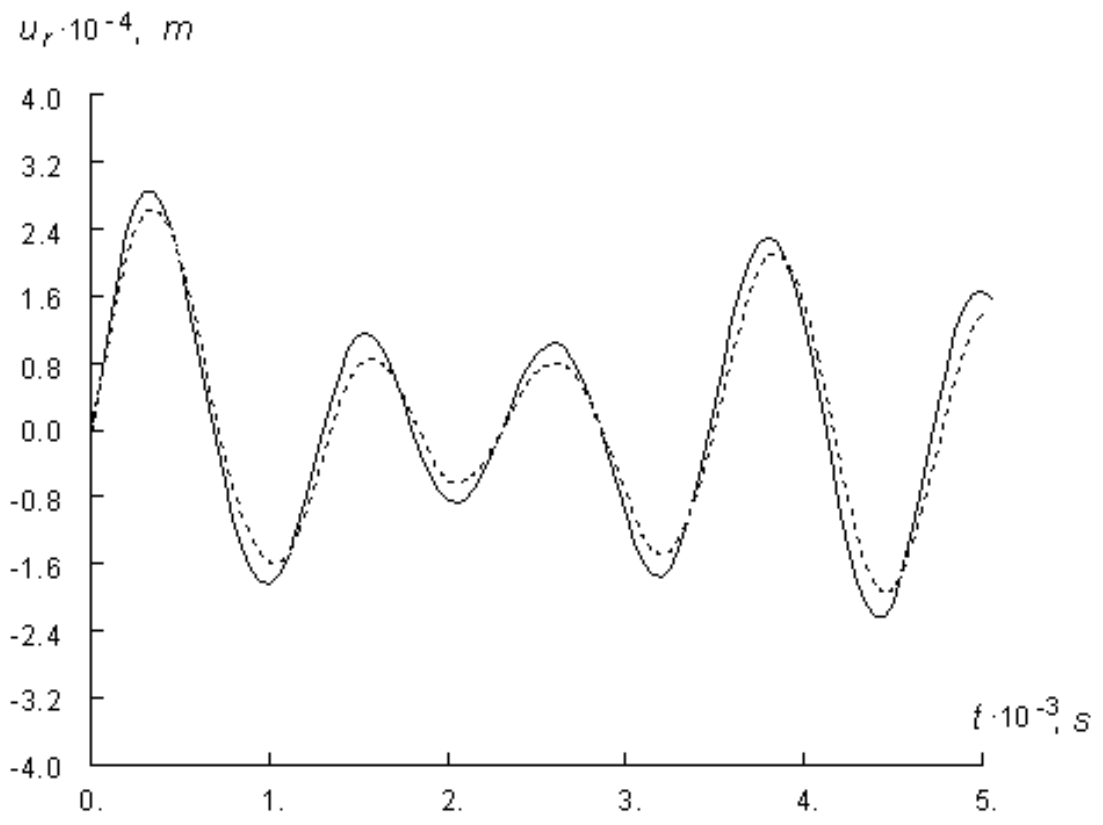

Figure 3: $\quad$ Time history of the radial displacement at point 2.

These figures illustrate the frequency slowness of those shell parts that are interacting with the fluid. Figures also demonstrate good agreement of results obtained by different methods. That testifies the reliability of the method and algorithm proposed. It would be noted that FEM analysis requires 3D modeling to solve this coupled problem.

\section{Conclusions}

The numerical procedure based on a coupling the finite element formulation and the boundary element method is developed for the analysis of free and forced vibrations of shells of revolution with an arbitrary meridian partially filled with the fluid. Integration by the fluid volume is reduced to integrals along the shell meridian and along the radius of the liquid free surface. It is the basic advantage of our method based on a combination of the boundary integral equations method and expansion into Fourier series. The governing integral equations for each harmonic have been obtained. According to the approach the free vibrations problem was reduced to consecutive definition of frequencies and modes of the shell vibrations in vacuum (the first stage), and to the solution of the mixed problems for Laplace equation (the second stage). The forced vibration problem includes the same steps involving the liquid added masses into equations of motion. Numerical investigations of natural frequencies and forced vibrations of the cylindrical tank filled with the incompressible fluid have been carried out. 


\section{Acknowledgements}

The authors gratefully acknowledge the sponsorship of this research by the Science and Technology Center in Ukraine in framework of the Project \#4624. Authors also would like to acknowledge our STCU Project collaborators Professors Carlos Brebbia and Eduard Ventsel for their constant support and interest to our research.

\section{References}

[1] Kubenko, V.D. \& Koval'chuk, P.S., Nonlinear problems of the dynamics of elastic shells partially filled with a liquid. International Journal of Applied Mechanics, 36(4), pp. 421-448, 2000.

[2] Amabili, M. \& Païdoussis, M. P., Review of studies on geometrically nonlinear vibrations and dynamics of circular cylindrical shells and panels, with and without fluid-structure interaction. Applied Mechanics Review, 56(4), pp. 349-381, 2003.

[3] Kumar, V.\& Ganesan, N., Dynamic analysis of conical shells conveying fluid. Journal of Sound and Vibration, 310(1-2), pp. 38-57, 2008.

[4] Malhotra, P. K., New method for seismic isolation of liquid-storage tanks. Journal of Earthquake Engineering and Structural Dynamics, 26(8), pp. 839-847, 1997.

[5] Zhang, Y.L., Gorman, D.G. \& Reese, J.M., Vibration of prestressed thin cylindrical shells conveying fluid. Thin-Walled Structures, 41, pp. 11031127, 2003.

[6] Chen, Y.H., Hwang, W.S. \& Ko, C.H., Numerical simulation of the threedimensional sloshing problem by boundary element method. Journal of the Chinese Institute of Engineers, 23(3), pp. 321-330, 2000.

[7] Brebbia, C.A., Telles, J.C.F. \& Wrobel, L.C. Boundary Element Techniques, Springer-Verlag: Berlin and New York, 1984. 